\title{
A framework for key considerations regarding point-of-care screening of newborns
}

\author{
Alex R. Kemper, MD, MPH ${ }^{1}$, Christopher A. Kus, MD, MPH², Robert J. Ostrander, $\mathrm{MD}^{3}$, \\ Anne Marie Comeau, $\mathrm{PhD}^{4}$, Coleen A. Boyle, $\mathrm{PhD}^{5}$, Denise Dougherty, $\mathrm{PhD}^{6}$, \\ Marie Y. Mann, MD, MPH${ }^{7}$, Jeffrey R. Botkin, MD, MPH ${ }^{8}$ and Nancy S. Green, MD ${ }^{9}$; on behalf \\ of the United States Secretary of Health and Human Services Advisory Committee \\ on Heritable Disorders in Newborns and Children
}

Newborn screening is performed under public health authority, with analysis carried out primarily by public health laboratories or other centralized laboratories. Increasingly, opportunities to improve infant health will arise from including screening tests that are completed at the birth centers instead of in centralized laboratories, constituting a significant shift for newborn screening. This report summarizes a framework developed by the US Secretary of Health and Human Services Advisory Committee on Heritable Disorders in Newborns and Children based on a series of meetings held during 2011 and 2012.
These meetings were for the purpose of evaluating whether conditions identifiable through point-of-care screening should be added to the recommended universal screening panel, and to identify key considerations for birth hospitals, public health agencies, and clinicians when point-of-care newborn screening is implemented.

Genet Med 2012:14(12):951-954

Key Words: health policy; neonatal screening; point-of-care systems; public systems

\section{INTRODUCTION}

In 2011, the US Secretary of Health and Human Services Advisory Committee on Heritable Disorders in Newborns and Children (SACHDNC) recommended that newborns be screened for critical congenital heart disease (CCHD) by pulse oximetry. This recommendation was made on the basis of evidence of the effectiveness of screening and the benefit of early intervention in improving child health. ${ }^{1}$ Other than screening for CCHD and congenital hearing loss, public health newborn screening (NBS) relies on centralized laboratories to analyze infant samples (i.e., dried-blood spots). At the time the CCHD recommendation was made, the SACHDNC recognized that state public health departments faced significant challenges in adopting screening recommendations that did not involve centralized laboratories.

The central issue is to determine the extent to which NBS that occurs outside of centralized laboratories should be overseen by NBS programs, as distinct from being conducted within the context of usual clinical care under the supervision of local health-care providers. The SACHDNC recognized the need to develop a standard approach to evaluating nursery-based NBS. Over a 1-year period, the SACHDNC developed a framework for considering nursery-based NBS that does not rely on centralized laboratories. This report describes the findings from this work, including key definitions, specific challenges, criteria for determining whether screening should be recommended, and the unique roles and responsibilities related to such screening activities.

\section{POINT-OF-CARE NBS}

Point-of-care (POC) testing includes tests administered and interpreted outside of a laboratory but close to the site of direct delivery of medical care to a patient. ${ }^{2}$ POC-NBS differs from the expected usual care provided by the health-care system in that the former includes universal access to follow-up diagnosis and treatment, aided by some degree of public health oversight. Usual care does not include these attributes, although it reflects current standards for care delivery, is supported by clinical guidelines produced by professional societies, and includes screening for a wide array of conditions (e.g., for conditions such as congenital hip dysplasia and visual impairment by physical examination even of newborns who appear to be well). Evidence-based recommendations for such clinical preventive approaches to care for newborns are available from sources such as Bright Futures and the United States Preventive Services Task Force. ${ }^{3,4}$ However, these components of routine care are not provided under public health authority, nor do public agencies provide direct oversight for performing screening, ensuring uniform quality of procedures, follow-up care, and reporting. A key feature of NBS, regardless of how it

\footnotetext{
${ }^{1}$ Department of Pediatrics, Duke University, Durham, North Carolina, USA; ${ }^{2}$ New York State Department of Health, Albany, New York, USA; ${ }^{3}$ alley View Family Practice Associates, Rushville, New York, USA; ${ }^{4}$ New England Newborn Screening Program and Department of Pediatrics, University of Massachusetts Medical School, Worcester, Massachusetts, USA; ${ }^{5}$ Centers for Disease Control and Prevention, Atlanta, Georgia, USA; ${ }^{6}$ Agency for Healthcare Research and Quality, Rockville, Maryland, USA; ${ }^{7}$ Health Resources and Services Administration, Rockville, Maryland, USA; ${ }^{8}$ Department of Pediatrics, University of Utah, Salt Lake City, Utah; USA; ${ }^{9}$ Department of Pediatrics, Columbia University Medical Center, New York City, New York, USA. Correspondence: Alex R. Kemper (alex.kemper@duke.edu)
} 
is implemented, is that it assures universal access to diagnostic and follow-up treatment services, and therefore plays an important role in eliminating disparities as regards management of clinical conditions of public health importance.

\section{CHALLENGES RELATED TO SCREENING FOR CONGENITAL HEARING LOSS AND CCHD}

In the 1990s, screening of newborns for the early identification of hearing loss began through hospital-based initiatives. By 2002, early detection and intervention programs for hearing loss were established as part of the public health system in all 50 states and the District of Columbia. ${ }^{5}$ Unlike NBS, which is based on the analysis of dried-blood spots within centralized laboratories, the screening test for congenital hearing loss is conducted in the newborn nursery and is based on an assessment of physiologic parameters (e.g., auditory evoked brainstem response, otoacoustic emissions) ${ }^{6}$ with diagnostic follow-up being available for infants with abnormal test results, who receive outpatient care by the age of 3 months. To implement the public health mandate for newborn hearing screening, birth hospitals acquired the necessary equipment; developed protocols to assure screening and communication of results to families, health-care providers, and state public health agencies; and trained their personnel in these protocols. ${ }^{7}$ Although nearly all newborns in the United States are screened for hearing loss before being discharged from the hospital, ${ }^{8}$ ensuring follow-up for infants with abnormal results remains a challenge. ${ }^{9,10}$ There has been no standardized approach to screening programs for hearing loss as regards the operation of the program and the responsibilities of various entities. In some states, the newborn hearing screening program assumes responsibility for monitoring hospital screening programs, follow-up of newborns who did not pass the screening, and tracking and reporting progress in these infants. In other states, the tracking of infants with abnormal results is primarily the responsibility of the institutions in which the testing was performed. In most states, the responsibility of public health authorities as regards NBS for hearing loss is primarily related to surveillance rather than individual case management, probably contributing to incomplete follow-up and reporting. ${ }^{9}$

As with congenital hearing loss, CCHD screening requires a physiologic test (i.e., pulse oximetry). However, unlike screening for congenital hearing loss, those with a positive result in the test for CCHD require urgent diagnostic testing before being discharged from the hospital. Important challenges as regards implementation include the need for a validated screening algorithm that takes into account local effects such as altitude differences between nurseries, as yet undeveloped quality assurance methods for the screening test, difficulties that some newborn nurseries face in obtaining off-site diagnostic testing capabilities, and the lack of established methods to report screening results and diagnostic follow-up data to the states' NBS programs.

\section{CRITERIA FOR POC-NBS}

In developing recommendations for POC-NBS, the SACHDNC will first evaluate the extent to which the following four criteria are met: (i) urgent treatment of the condition is required earlier than the feasible turnaround time for a public health laboratory; (ii) the screening is based on physiologic testing that requires the presence of the newborn at the time the results are generated; (iii) the public health impact of screening for early diagnosis and treatment is of sufficient importance; and (iv) universal screening and/or follow-up and treatment for the condition are not currently performed under standard clinical practice. The first two criteria establish the need for POC screening. The first criterion, namely, the need for urgent treatment and therefore for rapid turnaround of a test, is the strongest argument for POC screening. The second criterion, namely, the need for the presence of the newborn for a physiologic test (e.g., hearing screening), is a compelling but less crucial argument. The SACHDNC would not need to consider this criterion if the first were met. However, the SACHDNC would consider the need for the presence of the newborn for a physiologic test if there were compelling evidence that universal screening was not feasible outside of the newborn nursery. The third and fourth criteria establish the need for screening within a public health context. Both of these would need to be met before recommending the adoption of POC-NBS for the condition.

For conditions that meet these criteria, consideration for inclusion in the recommended universal screening panel should include an assessment of the feasibility of decentralized implementation, including not only the screening test but also the follow-up services. Before POC-NBS is recommended, it must be demonstrated that screening technology is readily available and can be standardized, that the screening protocol can feasibly be administered in the often chaotic newborn nursery setting without significant loss of clinical validity, and that appropriate follow-up diagnosis and care can be begun promptly for those with a positive test result. Ultimately, the decision about whether a condition meets the threshold for POC-NBS is predicated upon evidence that substantially better health outcomes are attainable if screening is performed under a public health mandate than can feasibly be obtained through usual clinical care.

\section{ROLES AND RESPONSIBILITIES IN POC-NBS}

Table 1 summarizes roles and responsibilities for public health programs and health-care providers across the components of POC-NBS. The extent to which public health agencies are directly involved in POC-NBS will depend on the legislation and regulations authorizing the particular screening test within each state. Factors that can help determine the degree of public health involvement include the risk of a missed affected case; the complexity of the screening procedure; whether the screening test is already a component of standard clinical care; the challenge of providing confirmatory diagnostic follow-up testing after an abnormal screening test result; and variability between sites as regards quality measures related to screening, diagnosis, and health outcomes. Regardless of the level of involvement, public 
Table 1 Summary of roles and responsibilities related to point-of-care newborn screening

\begin{tabular}{lll} 
Component & Public health agencies & Health-care providers \\
\hline Screening test & $\begin{array}{l}\text { Promote standard algorithm } \\
\text { Provide quality assurance } \\
\text { Train health-care providers } \\
\text { Provide educational material }\end{array}$ & $\begin{array}{l}\text { Adopt standard algorithm } \\
\text { Ensure all newborns are screened } \\
\text { Participate in quality assurance } \\
\text { Educate families } \\
\text { Document results } \\
\text { Share results with families }\end{array}$ \\
\hline Diagnostic evaluation & & $\begin{array}{l}\text { Ensure that those with an abnormal screening } \\
\text { result receive timely diagnostic evaluation }\end{array}$ \\
\hline Surveillance & Ensure the availability of diagnostic & Report results of screening and diagnostic \\
& evaluation & evaluation \\
\hline Long-term follow-up & Track results of screening and diagnostic & Provide long-term follow-up \\
\hline
\end{tabular}

health departments should, at a minimum, have roles in informing the public about new screening tests for conditions; facilitating standardized implementation of screening; participating in quality assurance; developing systems for diagnostic confirmation and follow-up; data collection on screening and diagnosis; and evaluating the extent to which the NBS is effective in improving child health. For some screening procedures or conditions, public health authorities may need to take a greater role in implementation and follow-up for POC screening than for others that can be undertaken within the context of routine care. For example, if screening for a condition requires special equipment or staff training, public health expertise may be needed for establishing standardized procedures and for evaluation of the quality of the implementation. Also, if availability of confirmatory diagnostic testing or treatment exists at only a limited number of sites, public health agencies could help facilitate transfer of patient care. For example, public health agencies might play a role in financing these rare but potentially costly activities. For some conditions, public health roles may be limited to educating the public and providers and standardizing the implementation.

\section{COSTS RELATED TO POC-NBS}

Central to the success of POC-NBS will be the availability of sufficient funding to meet the needs of a comprehensive screening program. Undoubtedly, this will require commitments from state legislatures as well as from payers. As with any screening program, the costs associated with POC-NBS include the costs of testing as well as follow-up. Important costs in addition to those associated with the actual administration of the screening test include those associated with purchase of screening equipment, start-up and continuation of hospital staff training, the development of information systems to track short- and long-term follow-up data, entering of results into these information systems, quality assurance monitoring, and program evaluation. The scientific evidence that underlies the need for screening, diagnosis, and treatment must provide a clear rationale for allocation of resources from clinical care and public health agencies to support POC-NBS programmatic activities.

\section{SUMMARY AND NEXT STEPS}

We expect that the number of conditions to be considered by the SACHDNC for inclusion in POC-NBS will continue to increase. It is likely that the continued development of rapid testing methodologies will yield a larger number of screening tests that can be done in the newborn nursery, transforming how NBS services are delivered. In contrast to usual clinical care, screening with public health oversight helps to ensure universal access and uptake of testing; high-quality standardized screening; coordinated follow-up with effective linkage to diagnosis, intervention, and family support; and surveillance. Expanding the use of electronic medical records and health information exchanges may help with documentation of screening and tracking of population health; such strategies will facilitate public health monitoring and evaluation of the delivery of POC-NBS services, from test administration through short- and long-term follow-up. The SACHDNC will continue to make recommendations for NBS based on the potential benefit of screening on health outcomes. At the same time, the SACHDNC will also consider feasibility in making recommendations regarding POC-NBS.

\section{ACKNOWLEDGMENTS}

The preparation of this report was supported by the logistics contract supporting the Secretary of Health and Human Services Advisory Committee on Heritable Disorders in Newborns and Children. The views expressed herein are solely those of the authors and do not necessarily reflect the views of the Secretary of the US Department of Health and Human Services or of the individual members of the Secretary's Advisory Committee on Heritable Disorders in Newborns and Children.

\section{DISCLOSURE}

The authors declared no conflict of interest.

\section{REFERENCES}

1. Kemper AR, Mahle WT, Martin GR, et al. Strategies for implementing screening for critical congenital heart disease. Pediatrics 2011; 128:e1259-e1267.

2. Price CP. Point of care testing. BMJ 2001;322:1285-1288.

3. Tanski S, Garfunkel LC, Duncan PM, Weitzman M (eds). Performing Preventive Services: A Bright Futures Handbook. American Academy of Pediatrics: Elk Grove Village, IL, 2010.

4. US Preventive Services Task Force. Recommendations. http://www uspreventiveservicestaskforce.org/recommendations.htm. Accessed 182011. 
5. White KR, Forsman I, Eichwald J, Munoz K. The evolution of early hearing detection and intervention programs in the United States. Semin Perinatol 2010:34:170-179.

6. Mehl AL, Thomson V. The Colorado Newborn Hearing Screening Project, 1992-1999: on the threshold of effective population-based universal newborn hearing screening. Pediatrics 2002;109:e7.

7. Joint Committee on Infant Hearing. Year 2007 position statement: principles and guidelines for early hearing detection and intervention programs. Pediatrics 2007;120:898-921.
8. Centers for Disease Control and Prevention. Hearing loss in children. http:// www.cdc.gov/ncbddd/hearingloss/index.html. Accessed 18 December 2011.

9. Russ SA, Hanna D, DesGeorges J, Forsman I. Improving follow-up to newborn hearing screening: a learning-collaborative experience. Pediatrics 2010;126(suppl 1):S59-S69.

10. Liu CL, Farrell J, MacNeil JR, Stone S, Barfield W. Evaluating loss to follow-up in newborn hearing screening in Massachusetts. Pediatrics 2008:121:e335-e343. 